\title{
On the seasonal dependence of relativistic electron fluxes
}

\author{
S. G. Kanekal ${ }^{1}$, D. N. Baker ${ }^{1}$, and R. L. McPherron ${ }^{2}$ \\ ${ }^{1}$ Laboratory for Atmospheric and Space Physics, University of Colorado, Boulder, CO, USA \\ ${ }^{2}$ Department of Earth and Space Science, University of California Los Angeles, Los Angeles, CA 90095-1567, USA
}

Received: 31 December 2008 - Revised: 27 April 2010 - Accepted: 28 April 2010 - Published: 10 May 2010

\begin{abstract}
The nature of the seasonal dependence of relativistic electron fluxes in the Earth's outer zone is investigated using 11 years of data from sensors onboard the SAMPEX spacecraft. It is found that, the relativistic electron fluxes show a strong semiannual modulation. However, the highest electron fluxes occur at times well away from the nominal equinoxes, lagging them by about 30 days. The time lag also shows a solar cycle phase dependence for the peak fluxes. The electron peak fluxes lag the vernal equinox by almost 60 days during the ascending phase of the solar cycle while the time lag near the autumnal equinox remains unchanged. The observed times of the peak electron fluxes during the descending phase of the solar cycle agrees most closely with the Russel-Mcpherron effect and less so with the equinoctial effect even after including propagation effects for finite solar wind speed. The observed times of the electron peaks are in disagreement with the axial effect. The asymmetrical response of the relativistic electrons during the ascending part of the solar cycle remains a puzzle.
\end{abstract}

Keywords. Magnetospheric physics (Energetic particles, precipitating; Energetic particles, trapped; Solar windmagnetosphere interactions)

\section{Introduction}

The dynamic nature of the relativistic electron populations in the Earth's outer zone is a result of balance between the processes of energization and loss. Electron energization in the outer zone may be due to particle transport or in-situ via wave particle interactions or a combination of the two. These processes are ultimately caused by high solar wind speeds (Paulikas and Blake, 1979; Baker et al., 1979) and strong

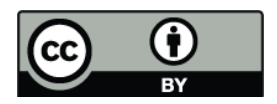

Correspondence to: S. G. Kanekal (shri.kanekal@ lasp.colorado.edu) southward component of the interplanetary magnetic field (Blake et al., 1997). The energization processes are thought to act upon a seed population of low energy electrons $(\approx$ hundreds of $\mathrm{keV}$ ) produced by substorms (Baker et al., 1998). Loss processes well away from the magnetopause are driven by magnetospheric waves of different kinds (see for example, Summers and Omura, 2007, and references therein). The energization and loss of electrons result in time variations of the outer zone electron fluxes ranging from tens of minutes to several days.

The relativistic electron fluxes in the radiation belts also vary on longer time scales showing a seasonal as well as a solar cycle dependence. The seasonal dependence is usually attributed to one (or a combination) of three mechanisms; the axial, the equinoctial and the Russell-McPherron effects. The axial effect explains the seasonal dependence as due to the Earth being at its highest helio-latitude during the equinoxes (Cortie, 1912) thereby increasing the exposure of the Earth's magnetosphere to high speed solar wind streams, prevalent at higher helio latitudes. The equinoctial effect depends upon the angle between the solar wind and the Earth's rotational axis, which is at its highest, i.e., $90^{\circ}$ at the equinoxes. The physical basis for the equinoctial effect is thought to be the increased geomagnetic activity due to Kelvin-Helmholtz type instabilities at the magnetopause (Boller and Stolov, 1970). The Russell-Mcpherron (RM) effect (Russell and McPherron, 1973) is a geometrical effect that explains the seasonal dependence in terms of increased negative southward component of the interplanetary magnetic field (IMF) in the geocentric solar magnetospheric (GSM) coordinate system. During equinoxes a larger component of the IMF is projected out of the ecliptic into the z-component in the GSM coordinate system.

The seasonal dependence of geomagnetic activity as measured by various indices has been examined in several studies which suggest a prominent role for the equinoctial effect and that it accounts for a substantial part of the observed seasonal

Published by Copernicus Publications on behalf of the European Geosciences Union. 
variations (see for example, Cliver et al., 2002, and references therein). For example, Svalgaard et al. (2002), suggest that up to $75 \%$ of the preponderance in occurrence frequency of "great" geomagnetic storms during the equinoxes may be due to the equinoctial effect. However, the relationship between geomagnetic storms and relativistic electrons is complex and a recent study by Reeves et al. (2003), has shown that only about half of geomagnetic storms lead to an enhancement in the electron fluxes. Therefore it follows that the seasonal variations of relativistic electrons may not be simply and straightforwardly attributed to the equinoctial effect, and merit a detailed study on their own.

Baker et al. (1999) have shown that the seasonal dependence of relativistic electron fluxes in the outer radiation belts is substantial, with the equinoctial fluxes being almost 3 times larger than the solstitial fluxes. They also found that the most pronounced seasonal variation among the interplanetary drivers of electron energization was in the southward component of the IMF. The southward component of the IMF is associated with substorms, which provide the seed population that is subsequently energized to relativistic energies by the solar wind. This led Baker et al. (1999) to suggest that both the RM and solar wind driven instabilities (BollerStolov effect, related to the equinoctial effect) were involved in the seasonal variation of relativistic electron fluxes. Another study by Li et al. (2001) also emphasized the role of solar wind and suggested that the equinoctial effect by itself could not account for the seasonal dependence of relativistic electron fluxes. Their observations showed that the peak times of the electron fluxes lagged the nominal equinoxes. They suggested that this lag may be due to the time required for radial transport of electrons from higher L-shells, which was assumed to be the dominant mechanism of energization.

In this work, we explore the seasonal dependence of relativistic electrons using data acquired during the period begining from 1993 to the end of 2002 which is nearly an entire solar cycle. We perform a superposed epoch analyses to determine the nature of the seasonal dependence for the entire period as well as during the descening and ascending parts of the solar cycles 22 and 23 . This study substantially extends earlier studies by probing the seasonal relativistic electron response during different phases of a solar cycle which are known to be dominated by different solar wind drivers and by covering the L-range comprising the entire outer zone.

\section{Data and analysis}

The electron data used in this study have been collected by the Proton Electron Telescope (PET) (Cook et al., 1993) onboard the Solar Anomalous Magnetospheric Particle EXplorer (SAMPEX) spacecraft. SAMPEX is in a low-Earth polar orbit at an altitude of $\approx 600 \mathrm{~km}$ and an inclination of $82^{\circ}$ (Baker et al., 1993) with an orbital period of $\approx 90 \mathrm{~min}$. The PET sensor comprises a stack of solid state detectors and observes electrons in the energy range from $\approx 0.4$ to $\approx 30 \mathrm{MeV}$. We use the daily averaged flux measurements of electrons in the energy range 2.0- to 6.0-MeV. Note that, due to the low Earth orbit of SAMPEX, the PET measures electrons that are stably trapped, or in either the bounce or the drift loss cone (Blake et al., 2001; Kanekal et al., 2001). We do not separate these populations and consider the full omnidirectional flux.

The electron flux measurements after correcting for dead time and mis-identification are sorted in nominal IGRF L values and binned in $0.1 \mathrm{~L}$ wide bins over the $\approx 15$ orbits that SAMPEX executes each day. The L-sorted flux values for each year are examined for "outlier" values which are replaced by interpolating from neighboring bins. The "outlier" values (due mostly to wrong dead time correction) are defined to be at least an order of magnitude higher than the average of the two neighbouring L-bins. The data are then box car smoothed over 3 L-bins.

In order to investigate the seasonal dependence of relativistic electron fluxes, we perform a superposed epoch analysis of the 11 years of measurements covering the years 1993 up to and including 2002. The zero epoch is simply the first day of year. We perform the superposed epoch analysis for 2 periods, the first covering the years 1993-1996 and the second covering the years 1997-2002. These time periods correspond to the descending and ascending part of the solar cycles 22 and 23, respectively. For each period, averages of logarithm of fluxes are calculated for each bin for each day. The $\log$ (fluxes) are used in order to minimize the skewing of superposed epoch analysis by large individual events. The superposed $\log$ (fluxes) are then smoothed with a 27-day box car smoothing, in order to remove the 27-day variations in the electron fluxes due to recurring high speed solar wind streams. Using the superposed data, we estimate the times of flux maxima of relativistic electrons for both the ascending and descending phases of solar cycles 22 and 23 .

The errors in determining the times of electron flux maxima arise mostly due to the 27-day box-car smoothing of the superposed $\log$ (fluxes). We estimate these errors by varying the smoothing interval from 21 to 31 days in steps of 2 days (i.e., 6 smoothing intervals) and calculating the electron peak times at each interval over a range of L-shells. For a given smoothing interval, the times of electron flux maxima $t_{i}(L)$, are obtained for $6 \mathrm{~L}$ values in the L-range of 3.5 to 4.5 at intervals of $0.2 \mathrm{~L}$. This L-range is chosen because it covers the location in $\mathrm{L}$ of the flux maximum. For each value of the smoothing interval, we obtain the mean, $\overline{t_{i}}$ and the standard deviation, $\sigma_{i}$ over the $\mathrm{L}$ range. we take as the "statistical" error, $\sigma_{\text {stat }}$ the standard deviation of the $6 \overline{t_{i}}$ and the average of the $6 \sigma_{i}$ as the "systematic error", $\sigma_{\text {syst }}$. To be conservative, we have used the average rather than the standard deviation to obtain $\sigma_{\text {syst }}$. The total error $\sigma_{\text {tot }}=\sqrt{\sigma_{\text {stat }}^{2}+\sigma_{\text {syst }}^{2}}$ is about 6(4) days for the vernal(autumnal) equinox for the descending phase (1993-1996) of the solar cycle. The 

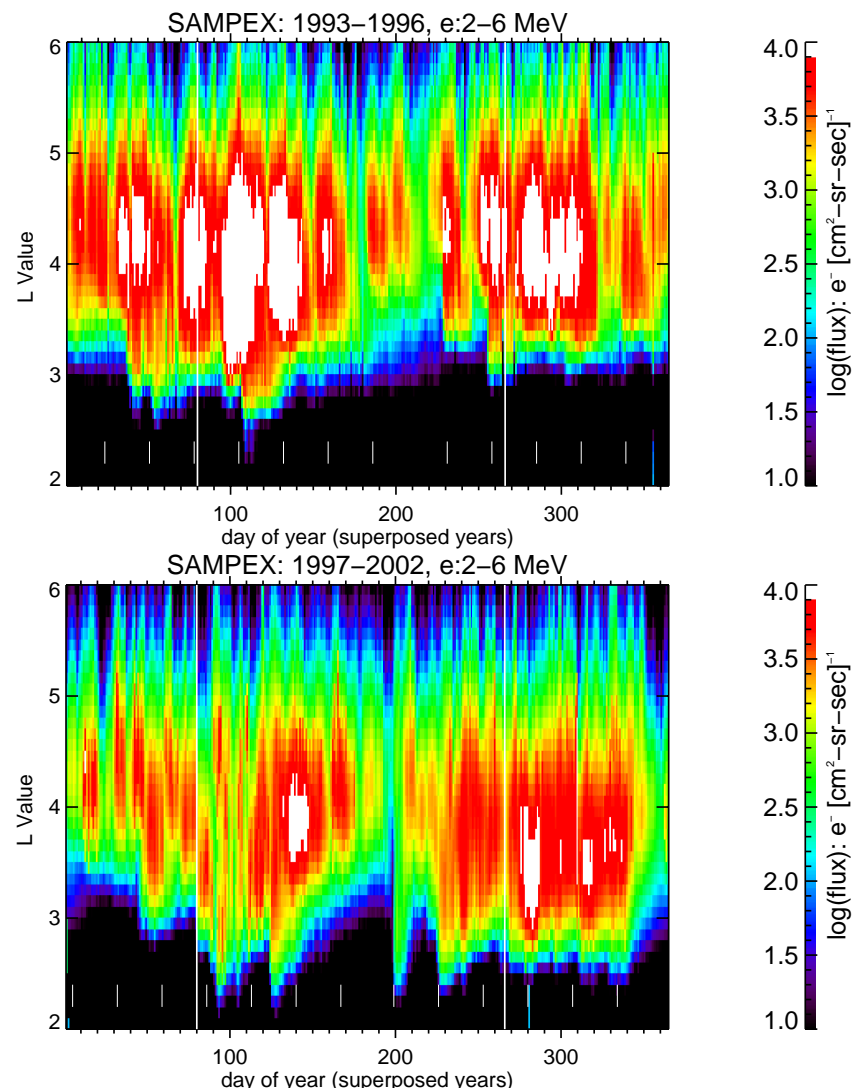

Fig. 1. (a) Annual superposed epoch analysis showing daily averaged 2- to 6-MeV electron $\log$ (fluxes) (1993-1996) during the descending part of solar cycle 22 . The times of 27 day intervals are shown by the short lines at the bottom part of the figure. (b) similar to (a) but for the ascending phase of solar cycle 23 (1996-2002).

corresponding $\sigma_{\text {tot }}$ for the ascending phase are 4 and 6 days for the vernal and autumnal equinoxes, respectively.

\section{Observations}

Figure 1a (Fig. 1b) shows the results of the superposed epoch analysis for the descending(ascending) part of the solar cycle 22(23). The plots show the logarithm of the 2- to 6-MeV electron fluxes, for two epoch periods: from year 1993 to 1996 and from year 1997 to 2002, respectively. The data shown are the unsmoothed daily averages, in order to specifically illustrate the 27 day recurring electron flux enhancements. The nominal vernal and autumnal equinoctial times are indicated by the white lines. The short white lines at the bottom part of each plot mark 27 day intervals. It is evident from Fig. 1a, that during the descending phase of the solar cycle, relativistic flux enhancements occur with a 27 day periodicity reflecting the periodicity of the high speed solar wind streams. These high speed streams emanate from longlived coronal holes which may last for a significant part of

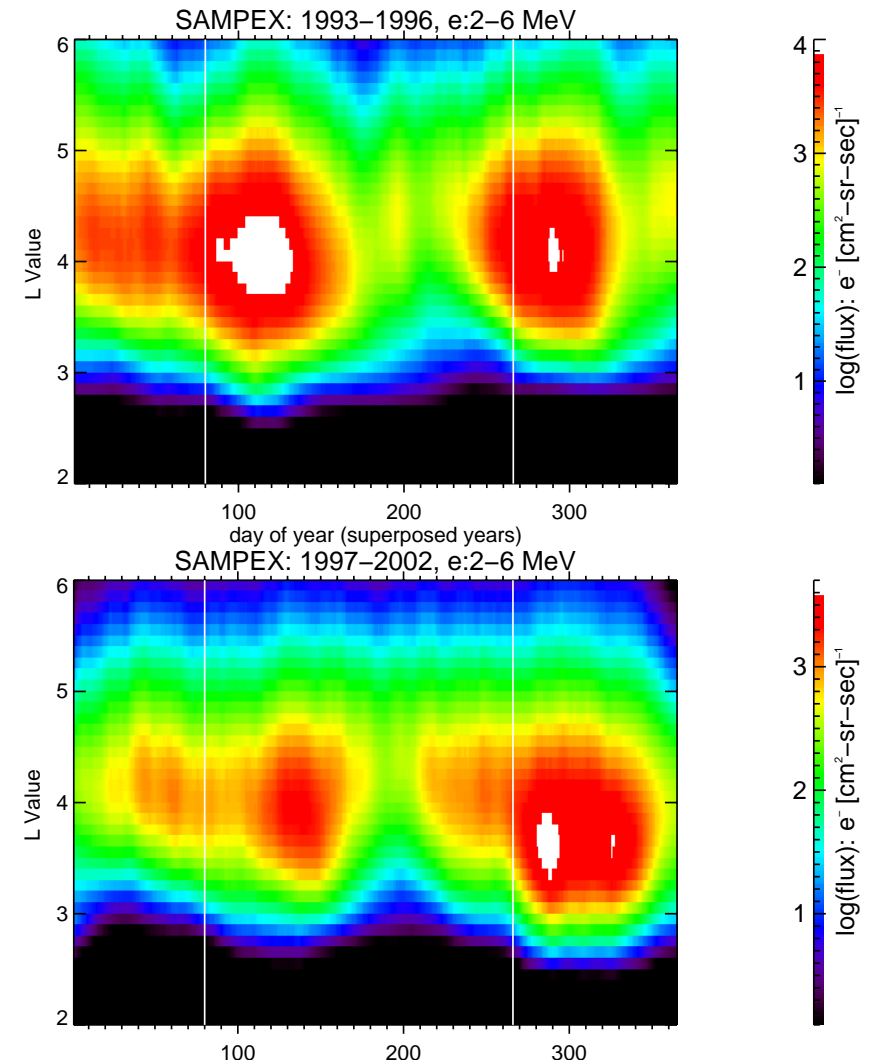

Fig. 2. (a) Annual superposed epoch analysis showing 27-day running box car averaged 2- to 6-MeV electron fluxes (1993-1996) during the descending part of solar cycle 22. (b) Similar to (a) but for the ascending phase of solar cycle 23 (1996-2002).

the solar cycle (Storini et al., 2006) and in 1993 a long-lived coronal hole that lasted almost 1.5 years was observed (Hofer and Storini, 2002). Furthermore, superposition by years for the period 1993-1996 corresponds nearly to a superposition by Carrington rotations. For this period the beginning of the years correspond closely, albeit not exactly, to Carrington rotations 1864, 1878, 1891, 1904 and 1918, respectively. Therefore it is not unexpected to observe the 27-day periodicity in a superposed epoch analysis as shown in Fig. 1. During the ascending phase (Fig. 1b) this periodicty is much less evident as coronal mass ejections which drive the flux enhancements occur sporadically.

The same data smoothed with a 27-day box-car averging method are shown in Fig. 2a and Fig. 2b. Note that the 27-day smoothing has been applied to yearly $\log$ (fluxes) before being superposed. As before the white lines indicate the times of the equinoxes. Two broad, distinct peaks in the relativistic electrons are clearly evident. The flux peaks are $\approx 2 \mathrm{~L}$ wide, centered around $L \approx 4$. These peaks in electron fluxes are displaced in time from the nominal equinoxes during both the descending and ascending phases of the solar cycle. Note that during the descending part of the solar cycle, the highest 


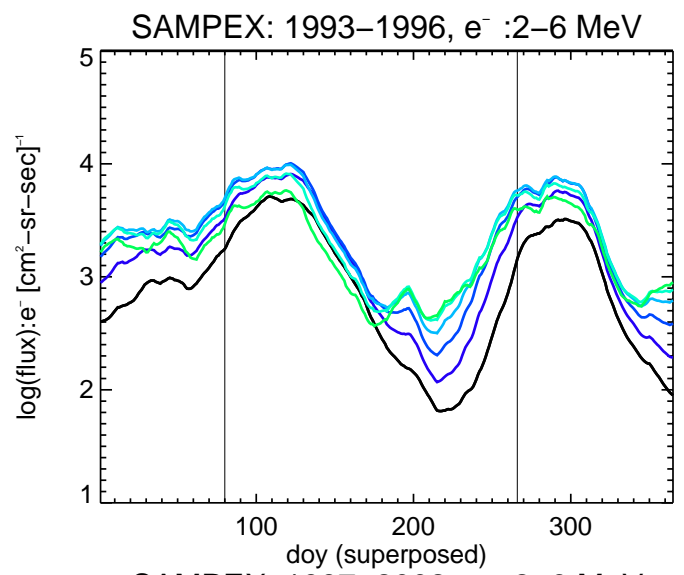

L (doy) $)_{\max }$ 3.5109297

3.7122292

3.9122291

4.1122290

4.3119290

4.5119285

vern. $118.8 \pm 5.0$

autm. $290.8 \pm 3.9$

SAMPEX: $1997-2002, \mathrm{e}^{-}: 2-6 \mathrm{MeV}$

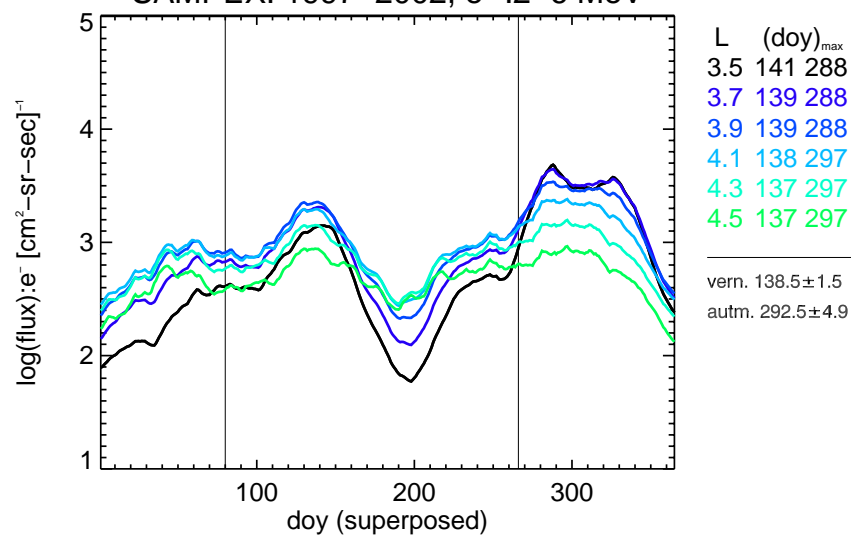

Fig. 3. (a) Superposed epoch fluxes of 27-day box car averaged 2- to 6-MeV electrons for L-values from 3.5 to 4.5 during the descending part of solar cycle 22 (1993-1996). The times of peak fluxes around the equinoctial times, their mean and standard deviations are indicated to the right. (b) Similar to (a) but for the ascending part of solar cycle 23 (1997-2002).

fluxes last much longer (especially about the vernal equinox) than the corresponding flux peaks during the ascending part. The longer duration of electron fluxes is due to recurring high speed solar wind streams emanating from well established coronal holes. During the ascending part of the solar cycle, it is the coronal mass ejections which predominate and drive the flux enhancements. They occur much more sporadically.

Figure $3 \mathrm{a}(3 \mathrm{~b})$ shows the superposed epoch fluxes at Lvalues centered about $L_{\max }$, the position in $L$ of the maximum flux, for the descending (ascending) part of the solar cycle 22(23). Both panels show the 27-day box-car smoothed daily-average fluxes. The nominal equinoctial times are indicated as in the previous figures. The superposed flux values and the corresponding peak times are shown for 6 selected $\mathrm{L}$ values around $L_{\max }$. The selected $\mathrm{L}$-values and the corresponding times of peak flux for each L-value are shown to the right. The mean and the standard deviation of the peak times are also indicated. It can be seen from Fig. 3a, that during the descending phase of the solar cycle the peak fluxes lag
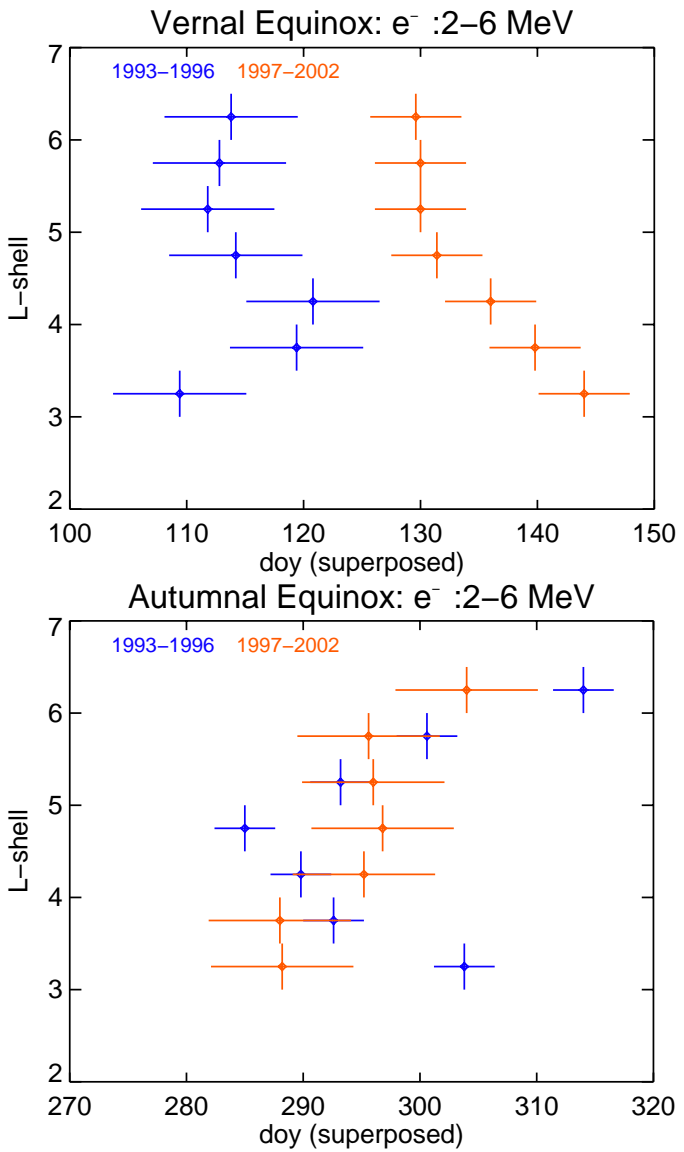

Fig. 4. (a) Observed times of peak fluxes for the 2- to 6-MeV electrons as a function of $L$ for the vernal equinox with the descending(ascending) phase shown in blue(red). (b) Similar to (a) but for the autumnal equinox.

the nominal equinoctial times by about 30 days with a mean spread of 2 days about the peak time. During the ascending part of the solar cycle, as is evident from Fig. 3b, the peak fluxes are displaced asymmetrically about the equinoxes. In this case, the peak electron fluxes lag the vernal equinox by almost 60 days but lag the autumnal equinox by only about 30 days.

Figure $4 \mathrm{a}(4 \mathrm{~b})$ shows the peak times of the electron fluxes as a function of L-value for the vernal (autumnal) equinox during both the ascending and descending parts of the solar cycle. The L-range shown covers the entire outer zone $(\approx 3$ to 6$)$. At each $\mathrm{L}$ value the peak position and the associated uncertainty are shown in blue(red) for the descending(ascending) phase of the solar cycle. It is evident that there is no strong systematic trend in the times of the peak position (within 1 to 2 sigma) as a function of $L$. Only for the vernal equinox during ascending phase, there appears to be a weak dependence of the peak times on $L$. This suggests that radial diffusion may not play a substantial role in determining the peak times for electrons in this energy range. 
Table 1. Observed times of peak fluxes for the 2- to 6-MeV electrons together with the expected times for the axial, RussellMcpherron and the equinoctial effects. Correcting for finite solar wind speed adds $\approx 4$ days to the nominal times for the equinoctial effect.

\begin{tabular}{lcc}
\hline \multirow{2}{*}{ Effect } & \multicolumn{2}{c}{ Peak activity time } \\
& Vernal & Autumnal \\
\hline Axial $^{\mathrm{a}}$ & 7 March & 9 September \\
Equinoctial $^{\dagger}$ & 21 March & 23 September \\
Russell - McPherron $^{\dagger}$ & 7 April & 11 October \\
Observations 1993-1996 & 29 April & 18 October \\
& \pm 6 days & \pm 4 days \\
Observations 1997-2002 & 19 May & 20 October \\
& \pm 4 days & \pm 6 days \\
\hline
\end{tabular}

a adapted from Cliver et al. (2002)

\section{Summary and conclusions}

Cliver et al. (2002) have suggested that the seasonal modulation of geomagnetic activity, as measured by the $a a$ index support the equinoctial hypothesis. In separate studies Cliver et al. (2001) and Cliver et al. (2000), examined the seasonal variation observed in other geomagnetic indices such as the Dst and am. They concluded that the storm component of the Dst index and the am index were also dominated by the equinoctial effect whose predictions agreed very well especially when finite solar wind speed was taken into account. However, Li et al. (2001) suggested that as far as relativistic electrons were concerned, the equinoctial effect alone was insufficient to explain the observations.

We have investigated the seasonal dependence of relativistic electrons across a wide range of L-shells covering both the descending and the ascending parts of the solar cycle. Our results show that the times of peak fluxes of relativistic electrons lag the nominal equinoxes significantly. The lag times are about 30 days during the descending phase of the solar cycle. The lag times are asymmetrical about the equinoxes during the ascending phase of the solar cycle, with the peak fluxes lagging the vernal equinox by almost 60 days with the lag time remaining the same (as that during the descending phase) at the autumnal equinox. Our observations also show that electron fluxes peak over a range of L-shells from $\approx 3.0-6.0$ (e.g., Fig. 4) almost simultaneously i.e., within our time resolution. We suggest therefore that radial transport may not explain fully the observed phase shift as suggested by Li et al. (2001). This is consistent with our earlier studies (Kanekal et al., 2001) using multi-satellite measurements, where we have reported on the global and coherent aspects of electron energization. Those measurements showed that electron fluxes rise fairly rapidly and across a large range of L-shells which suggests that in-situ rather than radial transport process may dominate electron energization.
As we mentioned before, the time lag from the equinoxes is significantly asymmetrical during the ascending part of the solar cycle. We have examined each of the 6 years of data during the ascending part for any systematic effect (e.g., any one particular year being predominant) and have found none. The observed asymmetry remains when we randomly exclude any single year of observations during the ascending phase. The asymmetry also persists when we limit the data to any three years between 1997 and 2002 (inclusive). None of the models suggest such an asymmetry. It is possible that the asymmetry may be an anomaly, as our data comprise only one period each of an ascending and a descending phase. However, our findings may also suggest that high speed streams and coronal mass ejections energize relativistic electron in different ways.

Table 1 lists the measured peak electron flux times and the expected peak activity due to the three proposed mechanisms. The predicted times for the equinoctial effect are shifted forward by $\approx 4$ days due to finite solar wind speed (average value of $\approx 440 \mathrm{~km} / \mathrm{s}$ ) (Li et al., 2001). The observations of the seasonal dependence of relativistic electrons presented here, suggest a more prominent role for the RussellMcpherron effect with the axial effect being the least important. While the equinoctial effect may be the dominant mechanism for the seasonal dependence of the geomagnetic activity, we suggest that it may be less so for the production of the relativistic electrons. It is well known that the southward component of the IMF plays a crucial role in determining which geomagnetic storms result in increased electron fluxes and which do not (Blake et al., 1997). We suggest that this may account for the dominance of the Russell-Mcpherron effect as far as relativistic electrons are concerned. The observed asymmetry of the peak fluxes during the ascending phase of the solar cycle is a puzzle that requires observations spanning multiple solar cycles before being established.

Acknowledgements. This work was supported by SAMPEX data grants. R. L. McPherron was supported by the NASA grants NNG04GA93G and NNX07AG16G.

Topical Editor I. A. Daglis thanks M. Hudson, S. Bourdarie, and another anonymous referee for their help in evaluating this paper.

\section{References}

Baker, D. N., Belian, R. D., Higbie, P. R., and Hones, E. W. J.: High-Energy Magnetospheric Protons and Their Dependence on Geomagnetic and Interplanetary Conditions, J. Geophys. Res., 84, 7138-7154, 1979.

Baker, D. N., Mason, G. M., Figueroa, O., Colon, G., Watzin, J. G., and Aleman, R. M.: An overview of the solar, anomalous, and magnetospheric particle explorer (SAMPEX) mission, IEEE Trans. Geosci. Rem. Sensing, 31, 531-541, 1993.

Baker, D. N., Pulkkinen, T. I., Li, X., Kanekal, S. G., Blake, J. B., Slesnick, R. S., Henderson, M. G., Reeves, G. D., Spence, H. E., and Rostoker, G.: Coronal mass ejections, magnetic clouds, and 
relativistic magnetospheric electron events: ISTP, J. Geophys. Res., 103, 17279-17292, doi:10.1029/97JA03329, 1998.

Baker, D. N., Kanekal, S. G., Pulkkinen, T. I., and Blake, J. B.: Equinoctial and solstitial averages of magnetospheric relativistic electrons: A strong semiannual modulation, Geophys. Res. Lett., 26, 3193-3196, doi:10.1029/1999GL003638, 1999.

Blake, J. B., Baker, D. N., Turner, N., Ogilvie, K. W., and Lepping, R. P.: Correlation of changes in the outer-zone relativistic-electron population with upstream solar wind and magnetic field measurements, Geophys. Res. Lett., 24, 927-930, doi:10.1029/97GL00859, 1997.

Blake, J. B., Selesnick, R. S., Baker, D. N., and Kanekal, S.: Studies of relativistic electron injection events in 1997 and 1998, J. Geophys. Res., 106, 19157-19 168, doi:10.1029/2000JA003039, 2001.

Boller, B. R. and Stolov, H. L.: Kelvin-Helmholtz instability and the semiannual variation of geomagnetic activity, J. Geophys. Res., 75, 6073-6084, 1970.

Cliver, E. W., kamide, Y., and Ling, A.: Mountains versus valleys:Semiannual variation of the geomagnetic activity, J. Geophys. Res., 105, 2413-2424, 2000.

Cliver, E. W., kamide, Y., Ling, A., and Yokoyama, N.: Semiannual variation of the geomagnetic $D_{s t}$ index: Evidence for a nonStorm component, J. Geophys. Res., 106, 21297-21304, 2001.

Cliver, E. W., kamide, Y., and Ling, A.: The semiannual variation of geomagnetic activity: phases and profiles for 130 years of aa data, J. Atmos. Solar Terr. Phys., 64, 47-53, 2002.

Cook, W. R., Cummings, A. C., Cummings, J. R., Garrard, T. L., Kecman, B., Mewaldt, R. A., Selesnick, R. S., Stone, E. C., Baker, D. N., von Rosenvinge, T. T., Blake, J. B., and Callis, L. B.: PET: A proton/electron telescope for studies of magnetospheric, solar, and galactic particles, IEEE Trans. Geosci. Rem. Sensing, 31, 565-571, doi:10.1109/36.225523, 1993.
Cortie, A. L.: Sunspots and terrestrial magnetic phenomena, 18981911; the cause of the annual variation in magnetic disturbances, Monthly Notices of the Royal Astron. Soc., 73, 52-+, 1912.

Hofer, M. Y. and Storini, M.: Peculiar features in the solar coronal hole occurrence, Solar Phys., 207, 1-10, 2002.

Kanekal, S. G., Baker, D. N., and Blake, J. B.: Multisatellite measurements of relativistic electron : Global coherence, J. Geophys. Res., 106, 29721-29732, doi:10.1029/2001JA000070, 2001.

Li, X., Baker, D. N., kanekal, S. G., Looper, M., and Temerin, M.: Long term measurements of radiation belts by SAMPEX and their variations, Geophys. Res. Lett., 28, 3827-3830, 10.1029/2001GL013586, 2001.

Paulikas, G. A. and Blake, J. B.: Effects of the solar wind on magnetospheric dynamics: Energetic electrons at the synchronous orbit, in: Quantitative Modeling of Magnetospheric Processes, edited by: Olsen, W. P., vol. 21, p. 180, AGU, Washington, D.C., 1979.

Reeves, G. D., McAdams, K. L., Friedel, R. H. W., and OB́rien, T. P.: Acceleration and loss of relativistic electrons during geomagnetic storms, Geophys. Res. Lett., 30, 36-1, doi:10.1029/2002GL016513, 2003.

Russell, C. T. and McPherron, R. L.: Semiannual variation of geomagnetic acivity, J. Geophys. Res., 78, 92, 1973.

Storini, M., Hofer, M., and Sykora, J.: Towards the understanding of coronal hole occurrence during the Schwabe cycle, Adv. Space Res., 38, 912-920, doi:10.1016/j.asr.2006.03.024, 2006.

Summers, D. and Omura, Y.: Ultra-relativistic acceleration of electrons in planetary magnetospheres, Geophys. Res. Lett., 34, 1143, doi:10.1029/2007GL032226, 2007.

Svalgaard, L., Cliver, E. W., and Ling, A. G.: The semiannual variation of great geomagnetic storms, Geophys. Res. Lett., 29, 160000-1, doi:029/2001GL014145, 2002. 